\title{
EW corrections on top-quark pair production: the impact of the photon PDF
}

\author{
loannis Tsinikos * \\ Centre for Cosmology, Particle Physics and Phenomenology (CP3), \\ Université Catholique de Louvain, Chemin du Cyclotron 2, B-1348 Louvain-la-Neuve, Belgium \\ E-mail: ioannis.tsinikos@uclouvain.be
}

In this proceeding we present the combination of NNLO QCD and NLO EW corrections to $t \bar{t}$ distributions, focusing on the effect of the photon PDF. We compare the LUXQED and NNPDF3.0QED PDF sets for specific distributions. We further discuss the effect of the EW corrections with respect to the pure QCD ones including scale and PDF uncertainties for both these PDF sets. We focus on the top-quark pair invariant mass, top-quark transverse momentum for $13 \mathrm{TeV}$ and on the top-quark pair rapidity for $8 \mathrm{TeV}$.

XXV International Workshop on Deep-Inelastic Scattering and Related Subjects 3-7 April 2017

University of Birmingham, UK

\footnotetext{
* Speaker. Preprint: CP3-17-22
} 


\section{Introduction}

The results discussed in this proceeding are part of what is presented in [1]. The $t \bar{t}$ observables have been calculated including the QCD corrections beyond the NLO [2-16]. The new run of LHC at $13 \mathrm{TeV}$ will decrease the experimental uncertainties and furthermore there is a reported tension between theory and experiment in the top-quark $p_{T}$ distribution at $8 \mathrm{TeV}[17,18]$. Furthermore, many studies on the NLO EW corrections to $t \bar{t}$ production are already available [19-30]. Having the complete NLO EW corrections requires the photon-induced contributions. On top of that these contributions may accidentally compensate part of the Sudakov suppression rendered by the virtual EW corrections. The PDF sets that include the photon PDF are the MRST2004QED [31], the NNPDF2.3QED [32], the APFEL_NN2.3QED [33,34], the CT14QED [35] and the more recent NNPDF3.0QED [36] and LUXQED [37]. Comparisons between the NNPDF2.3QED and CT14QED PDF sets in the $t \bar{t}$ process are presented in [38,39]. Similar differences have been pointed out in dilepton final states $[40,41]$ between the NNPDFQED and the CT14QED, LUXQED PDF sets.

\section{Calculation framework}

In this work we realise a NNLO QCD + NLO EW calculation for $t \bar{t}$ distributions. For the NNLO QCD part we use the calculational techniques of [5] and for the NLO EW part we use an extension of the MADGRAPH5_AMC@NLO framework [42] already validated in [43-45]. In figure 1 we show a pictorial representation of the perturbative orders considered in the calculation.

LO
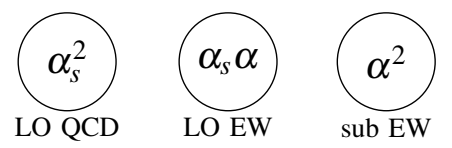

NLO
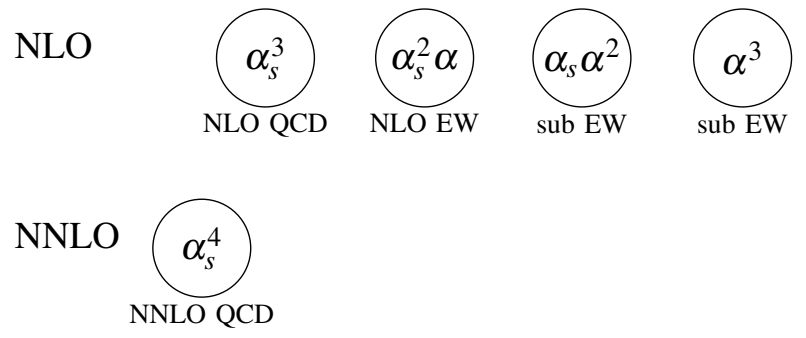

Figure 1: Perturbative orders included in the calculation.

The inclusion of the NNLO QCD corrections reduce significantly the scale uncertainties. Therefore, in contrast to what is done in [38], we include the subleading $\alpha^{2}, \alpha_{s} \alpha^{2}, \alpha^{3}$ orders, which we denote as sub EW. For any observable $\Sigma$ we define the QCD, EW and QCD+EW orders as shown in equation 2.1.

$$
\begin{aligned}
& \Sigma_{\mathrm{QCD}} \equiv \Sigma_{\mathrm{LO} \mathrm{QCD}}+\Sigma_{\mathrm{NLO}} \mathrm{QCD}+\Sigma_{\mathrm{NNLO}} \mathrm{QCD} \\
& \Sigma_{\mathrm{EW}} \equiv \Sigma_{\mathrm{LO} \mathrm{EW}}+\Sigma_{\mathrm{NLO} \mathrm{EW}}+\Sigma_{\text {sub EW }} \\
& \Sigma_{\mathrm{QCD}+\mathrm{EW}} \equiv \Sigma_{\mathrm{QCD}}+\Sigma_{\mathrm{EW}}
\end{aligned}
$$


We have used the 5-flavour scheme for the calculation and the EW parameters are defined in the $G_{\mu}$-scheme. In equation 2.2 we show the input parameters of the calculation.

$$
\begin{aligned}
m_{t} & =173.3 \mathrm{GeV}, m_{H}=125.09 \mathrm{GeV}, m_{W}=80.385 \mathrm{GeV}, m_{Z}=91.1876 \mathrm{GeV}, \\
G_{\mu} & =1.1663787 \cdot 10^{-5} \mathrm{GeV}^{-2}, \\
\mu & =\frac{m_{T, t}}{2} \text { for the } p_{T}(t) \text { distribution, } \mu=\frac{m_{T, \bar{t}}}{2} \text { for the } p_{T}(\bar{t}) \text { distribution, } \\
\mu & =\frac{H_{T}}{4}=\frac{1}{4}\left(m_{T, t}+m_{T, \bar{t}}\right) \text { for the } m(t \bar{t}), y_{\text {avt }}, y(t \bar{t}) \text { distributions. }
\end{aligned}
$$

The observables $p_{T, \text { avt }}, y_{\text {avt }}$ are the average of the two top $p_{T}, y$ distributions respectively. The scale choice for each observable is based on the principle of the fastest convergence and is taken from [5]. For the theoretical uncertainties we use the 7-point variation within the interval $\{\mu / 2<$ $\left.\mu_{f}, \mu_{r}<2 \mu\right\}$. In the following section we focus on the comparison between the NNPDF3.0QED and LUXQED PDF sets at $13 \mathrm{TeV}$.
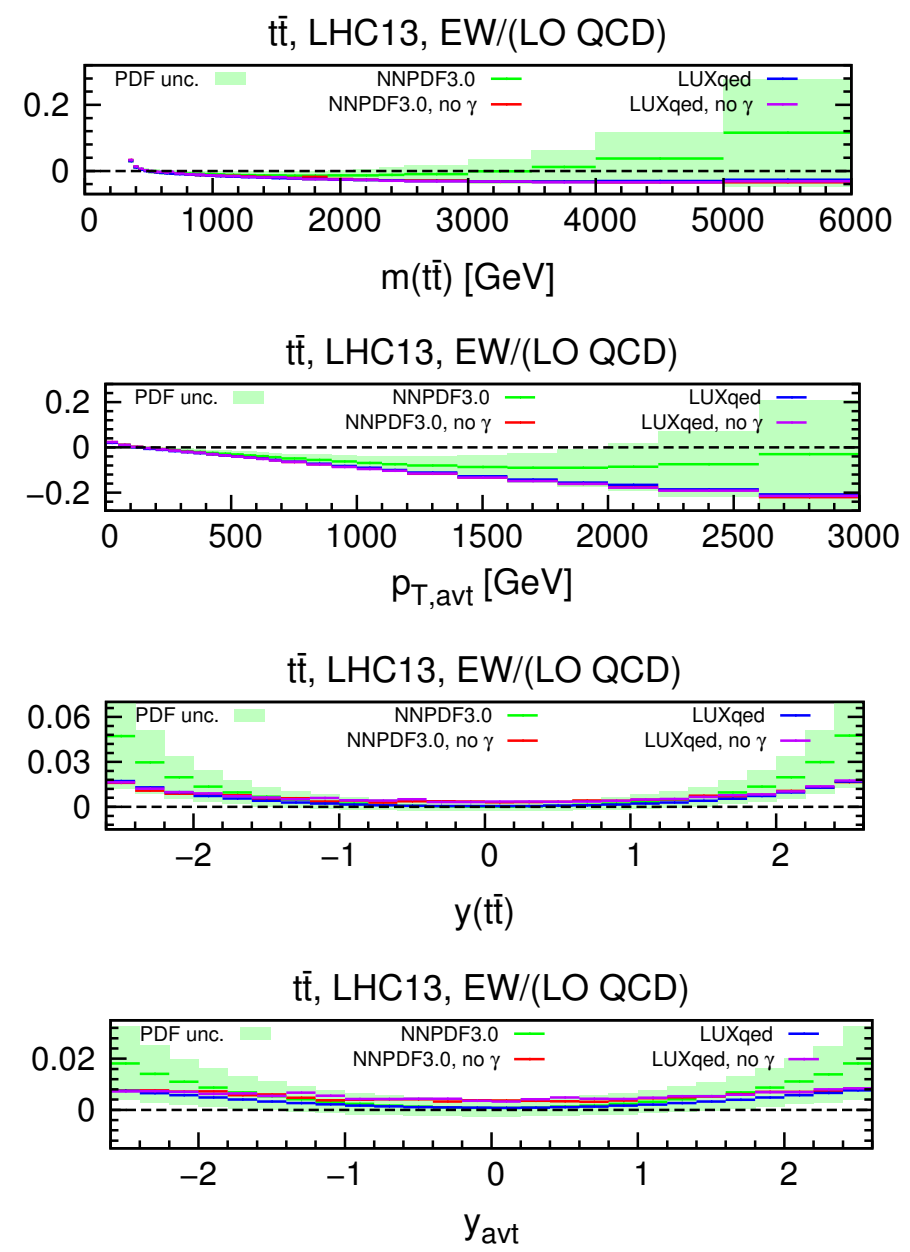

Figure 2: The impact of the photon PDF for the $m(t \bar{t}), p_{T, \text { avt }}, y_{\text {avt }}, y_{t \bar{t}}$ distributions for the NNPDF3.0QED and the LUXQED PDF sets. 


\section{Results}

We first compare the two PDF sets for all the differential distributions and for a more detailed discussion we restrict ourselves to the $m(t \bar{t}), p_{T, \text { avt }}$ and $y(t \bar{t})$ ones. In figure 2 we present the ratio EW/(LO QCD). For both the PDF sets considered we further artificially set the photon PDF equal to zero in order to isolate the effect of the photon-induced contributions. In these distributions we can see that the impact of the photon PDF is large for the NNPDF3.0QED PDF set, accompanied with large uncertainties. On the other hand this effect is negligible for the LUXQED PDF set. In all cases the LUXQED is equivalent to the NNPDF3.0QED - no $\gamma$ and they are in agreement within the uncertainties.
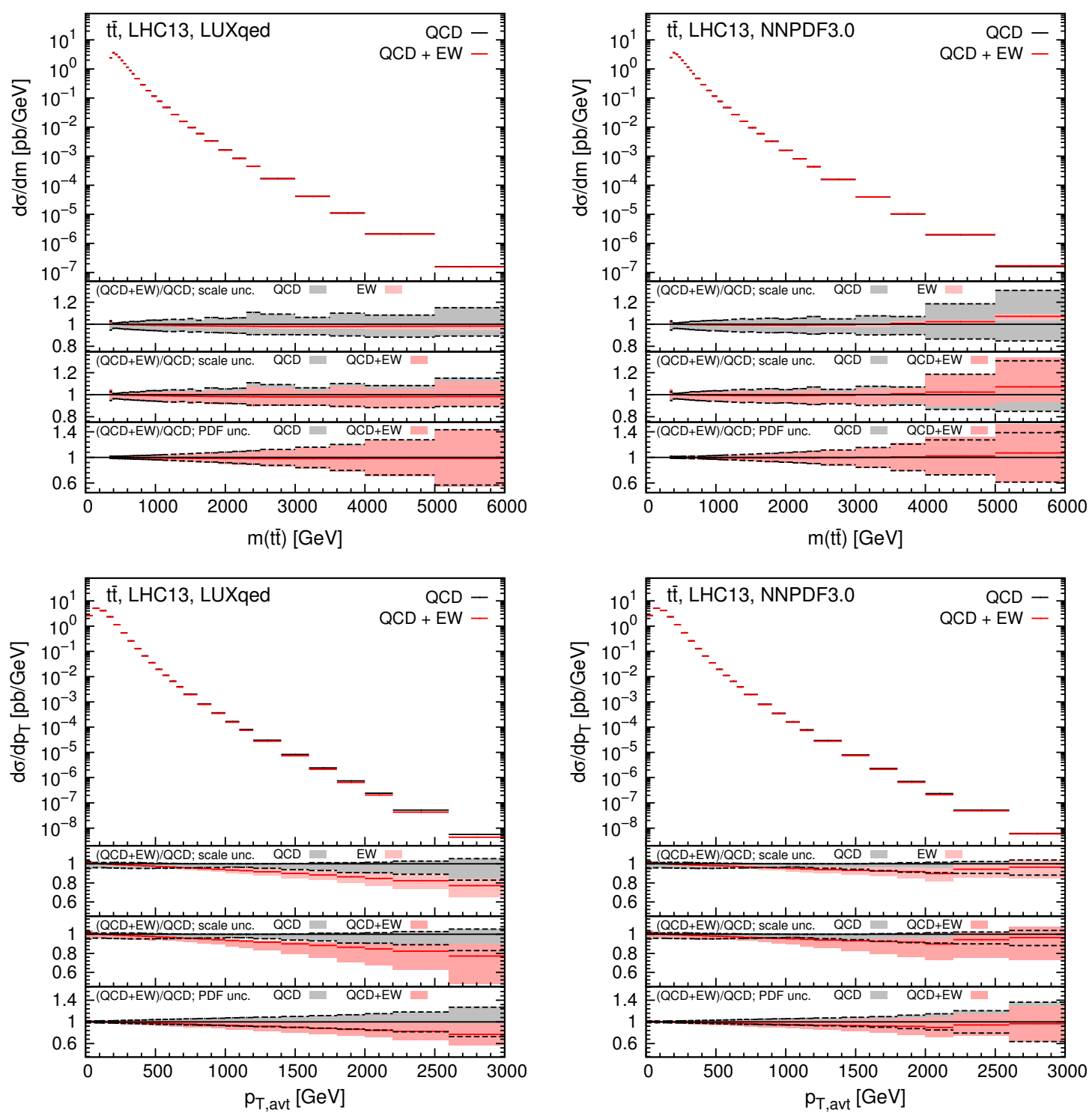

Figure 3: The $m(t \bar{t})$ (top) and $p_{T, \text { avt }}$ (bottom) distributions at $13 \mathrm{TeV}$. The format of the plots is explained in the text. 
Focusing on specific differential distributions at $13 \mathrm{TeV}$ we show the $m(t \bar{t})$ and $p_{T \text {,avt }}$ results (fig. 3). The format of the plots is the following. In the main panel we show the distributions of the QCD (black) and QCD+EW (red) according to the definitions of equation 2.1. In the three insets we show the ratio $(\mathrm{QCD}+\mathrm{EW}) / \mathrm{QCD}$. In the first inset we put the scale uncertainties of the QCD and the EW separately and in the second inset the QCD and the QCD+EW ones. In the third inset we show the PDF uncertainties of the QCD and of the QCD+EW. On the left plot of fig 3 we have the results with the LUXQED and on the right plot the ones with the NNPDF3.0QED. Starting with the $m(t \bar{t})$ distribution and looking at the first inset, we can see that in both cases the EW corrections are small. One can also notice in the third inset that at the high $m(t \bar{t})$ region the dominant theory uncertainty is coming from the PDFs rather than from the scale variation. In the $p_{T \text {,avt }}$ distribution the situation is different. In the LUXQED case, the EW corrections reach the $\sim-20 \%$ at the tail of the distribution due to the Sudakov suppression. On top of that the $\mathrm{QCD}+\mathrm{EW}$ result deviates from the pure QCD one already at the $\sim 1 \mathrm{TeV}$ range outside the QCD scale uncertainty band. Moving to the right plot we see that for the NNPDF3.0QED case, the photon-induced contributions compensate the negative Sudakov logarithms and reduce in absolute value the EW corrections.
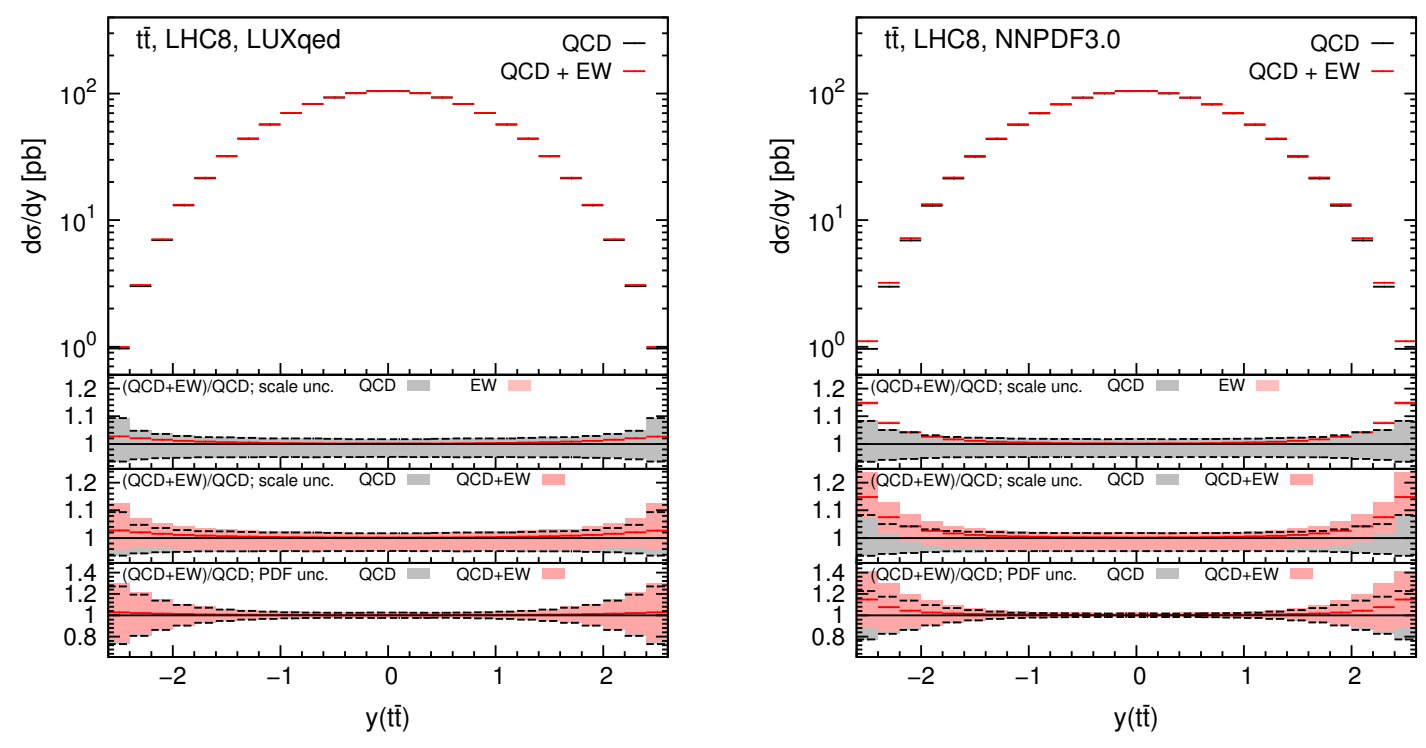

Figure 4: The $y(t \bar{t})$ distribution at $8 \mathrm{TeV}$. The format of the plot is explained in the text.

For the $y(t \bar{t})$ distribution we move to $8 \mathrm{TeV}$ (fig 4). The reason is that with lower $\sqrt{s}$ we probe higher Bjorken $x$ values, which are more sensitive to the photon-indiced contributions. In the right plot of figure 4 we see that for the NNPDF3.0QED case there is a $\sim 15 \%$ effect due to the photon $\mathrm{PDF}$ at the large rapidity regions.

\section{Conclusions}

In this proceeding we show part of the work presented in [1]. We restrict ourselves to the additive combination of the NNLO QCD and NLO EW corrections to $t \bar{t}$ distributions and focus 
on the impact of the photon-induced contributions. We compare at differential level the results obtained with the LUXQED and NNPDF3.0QED PDF sets. The results with the former show a negligible effect of the photon-induced contributions being in the lower limit of the uncertainty band of the latter. The NNPDF3.0QED PDF set predicts a large photon PDF effect with large uncertainties. The EW corrections are small for both the PDF sets in the $m(t \bar{t})$ distribution, but they are large and PDF set dependent in the case of the $p_{T, \text { avt }}$ distribution at $13 \mathrm{TeV}$ and the $y(t \bar{t})$ distribution at $8 \mathrm{TeV}$.

\section{Acknowledgments}

I would like to thank M. Czakon, D. Heymes, A. Mitov, D. Pagani and M. Zaro for their collaboration on this work. My participation to this workshop was supported by the F.R.S.-FNRS "Fonds de la Recherche Scientifique" (Belgium).

\section{References}

[1] M. Czakon, D. Heymes, A. Mitov, D. Pagani, I. Tsinikos and M. Zaro, Top-pair production at the LHC through NNLO QCD and NLO EW, 1705.04105.

[2] M. Czakon, M. L. Mangano, A. Mitov and J. Rojo, Constraints on the gluon PDF from top quark pair production at hadron colliders, JHEP 07 (2013) 167, [1303 . 7215].

[3] M. Czakon, P. Fiedler and A. Mitov, Total Top-Quark Pair-Production Cross Section at Hadron Colliders Through $O\left(\alpha_{S}^{4}\right)$, Phys. Rev. Lett. 110 (2013) 252004, [1303.6254].

[4] M. Czakon, D. Heymes and A. Mitov, High-precision differential predictions for top-quark pairs at the LHC, Phys. Rev. Lett. 116 (2016) 082003, [1511.00549].

[5] M. Czakon, D. Heymes and A. Mitov, Dynamical scales for multi-TeV top-pair production at the LHC, JHEP 04 (2017) 071, [1606.03350].

[6] S. Moch and P. Uwer, Theoretical status and prospects for top-quark pair production at hadron colliders, Phys. Rev. D78 (2008) 034003, [0 804 . 1476].

[7] N. Kidonakis, Two-loop soft anomalous dimensions and NNLL resummation for heavy quark production, Phys. Rev. Lett. 102 (2009) 232003, [0903.2561].

[8] M. Czakon, A. Mitov and G. F. Sterman, Threshold Resummation for Top-Pair Hadroproduction to Next-to-Next-to-Leading Log, Phys. Rev. D80 (2009) 074017, [0 907.1790$].$

[9] V. Ahrens, A. Ferroglia, M. Neubert, B. D. Pecjak and L. L. Yang, Renormalization-Group Improved Predictions for Top-Quark Pair Production at Hadron Colliders, JHEP 09 (2010) 097, [1003.5827].

[10] M. Beneke, P. Falgari and C. Schwinn, Threshold resummation for pair production of coloured heavy (s)particles at hadron colliders, Nucl. Phys. B842 (2011) 414-474, [1007.5414].

[11] N. Kidonakis, Next-to-next-to-leading soft-gluon corrections for the top quark cross section and transverse momentum distribution, Phys. Rev. D82 (2010) 114030, [1 009.4935$].$

[12] V. Ahrens, A. Ferroglia, M. Neubert, B. D. Pecjak and L.-L. Yang, RG-improved single-particle

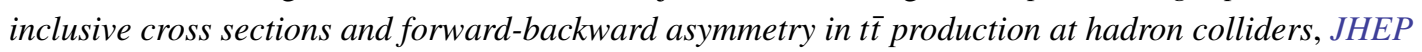
09 (2011) 070, [1103.0550]. 
[13] V. Ahrens, A. Ferroglia, M. Neubert, B. D. Pecjak and L. L. Yang, Precision predictions for the $t+t$ (bar) production cross section at hadron colliders, Phys. Lett. B703 (2011) 135-141, [1105.5824].

[14] M. Beneke, P. Falgari, S. Klein and C. Schwinn, Hadronic top-quark pair production with NNLL threshold resummation, Nucl. Phys. B855 (2012) 695-741, [1109.1536].

[15] M. Cacciari, M. Czakon, M. Mangano, A. Mitov and P. Nason, Top-pair production at hadron colliders with next-to-next-to-leading logarithmic soft-gluon resummation, Phys. Lett. B710 (2012) 612-622, [1111.5869].

[16] B. D. Pecjak, D. J. Scott, X. Wang and L. L. Yang, Resummed differential cross sections for top-quark pairs at the LHC, 1601.07020.

[17] CMS collaboration, V. Khachatryan et al., Measurement of the differential cross section for top quark pair production in pp collisions at $\sqrt{s}=8 \mathrm{TeV}$, Eur. Phys. J. C75 (2015) 542, [1505. 04480 ].

[18] ATLAS collaboration, G. Aad et al., Measurements of top-quark pair differential cross-sections in the lepton+jets channel in pp collisions at $\sqrt{s}=8 \mathrm{TeV}$ using the ATLAS detector, 1511.04716.

[19] W. Beenakker, A. Denner, W. Hollik, R. Mertig, T. Sack and D. Wackeroth, Electroweak one loop contributions to top pair production in hadron colliders, Nucl. Phys. B411 (1994) 343-380.

[20] J. H. Kühn, A. Scharf and P. Uwer, Electroweak effects in top-quark pair production at hadron colliders, Eur. Phys. J. C51 (2007) 37-53, [hep-ph/0610335].

[21] J. H. Kühn, A. Scharf and P. Uwer, Weak Interactions in Top-Quark Pair Production at Hadron Colliders: An Update, Phys. Rev. D91 (2015) 014020, [1305. 5773].

[22] W. Bernreuther, M. Fuecker and Z. G. Si, Mixed QCD and weak corrections to top quark pair production at hadron colliders, Phys. Lett. B633 (2006) 54-60, [hep-ph/ 0508091 ].

[23] J. M. Campbell, D. Wackeroth and J. Zhou, Study of weak corrections to Drell-Yan, top-quark pair, and dijet production at high energies with MCFM, Phys. Rev. D94 (2016) 093009, [1 608 . 0335 6].

[24] W. Hollik and D. Pagani, The electroweak contribution to the top quark forward-backward asymmetry at the Tevatron, Phys. Rev. D84 (2011) 093003, [1107.2606].

[25] J. H. Kühn and G. Rodrigo, Charge asymmetries of top quarks at hadron colliders revisited, JHEP 01 (2012) 063, [1109.6830].

[26] A. V. Manohar and M. Trott, Electroweak Sudakov Corrections and the Top Quark Forward-Backward Asymmetry, Phys. Lett. B711 (2012) 313-316, [1201. 3926].

[27] W. Bernreuther and Z.-G. Si, Top quark and leptonic charge asymmetries for the Tevatron and LHC, Phys. Rev. D86 (2012) 034026, [1205.6580].

[28] W. Bernreuther and Z.-G. Si, Distributions and correlations for top quark pair production and decay at the Tevatron and LHC., Nucl. Phys. B837 (2010) 90-121, [1003.3926].

[29] A. Denner and M. Pellen, NLO electroweak corrections to off-shell top-antitop production with leptonic decays at the LHC, JHEP 08 (2016) 155, [1607.05571].

[30] W. Hollik and M. Kollar, NLO QED contributions to top-pair production at hadron collider, Phys. Rev. D77 (2008) 014008, [0 708 . 1697].

[31] A. D. Martin, R. G. Roberts, W. J. Stirling and R. S. Thorne, Parton distributions incorporating QED contributions, Eur. Phys. J. C39 (2005) 155-161, [hep-ph/ 0411040 ]. 
[32] NNPDF collaboration, R. D. Ball, V. Bertone, S. Carrazza, L. Del Debbio, S. Forte, A. Guffanti et al., Parton distributions with QED corrections, Nucl. Phys. B877 (2013) 290-320, [1308 . 0598 ].

[33] V. Bertone, S. Carrazza and J. Rojo, APFEL: A PDF Evolution Library with QED corrections, Comput. Phys. Commun. 185 (2014) 1647-1668, [1310.1394].

[34] V. Bertone, S. Carrazza, D. Pagani and M. Zaro, On the Impact of Lepton PDFs, JHEP 11 (2015) 194, [1508.07002].

[35] C. Schmidt, J. Pumplin, D. Stump and C. P. Yuan, CT14QED parton distribution functions from isolated photon production in deep inelastic scattering, Phys. Rev. D93 (2016) 114015, [1509.02905].

[36] NNPDF collaboration, R. D. Ball et al., Parton distributions for the LHC Run II, JHEP 04 (2015) 040, [1410.8849].

[37] A. Manohar, P. Nason, G. P. Salam and G. Zanderighi, How bright is the proton? A precise determination of the photon parton distribution function, Phys. Rev. Lett. 117 (2016) 242002 , [1607.04266].

[38] D. Pagani, I. Tsinikos and M. Zaro, The impact of the photon PDF and electroweak corrections on $t \bar{t}$ distributions, 1606.01915.

[39] I. Tsinikos, EW corrections to t $\bar{t}$ distributions: the photon PDF effect, in 9th International Workshop on Top Quark Physics (TOP 2016) Olomouc, Czech Republic, September 19-23, 2016, 2016. 1611.08234.

[40] E. Accomando, J. Fiaschi, F. Hautmann, S. Moretti and C. H. Shepherd-Themistocleous, The effect of real and virtual photons in the di-lepton channel at the LHC, Phys. Lett. B770 (2017) 1-7, [1612.08168].

[41] E. Accomando, J. Fiaschi, F. Hautmann, S. Moretti and C. H. Shepherd-Themistocleous, Real and virtual photons effects in di-lepton production at the LHC, in 21st Rencontres de Physique de La Vallée d'Aoste: Rencontres de La Thuile (La Thuile) La Thuile, Aosta, Italy, March 5-11, 2017, 2017. 1704.08587.

[42] J. Alwall, R. Frederix, S. Frixione, V. Hirschi, F. Maltoni, O. Mattelaer et al., The automated computation of tree-level and next-to-leading order differential cross sections, and their matching to parton shower simulations, JHEP 07 (2014) 079, [1405.0301].

[43] S. Frixione, V. Hirschi, D. Pagani, H. S. Shao and M. Zaro, Electroweak and QCD corrections to top-pair hadroproduction in association with heavy bosons, JHEP 06 (2015) 184, [15 04.03446 ].

[44] J. R. Andersen et al., Les Houches 2015: Physics at TeV Colliders Standard Model Working Group Report, in 9th Les Houches Workshop on Physics at TeV Colliders (PhysTeV 2015) Les Houches, France, June 1-19, 2015, 2016. 1605.04692.

[45] R. Frederix, S. Frixione, V. Hirschi, D. Pagani, H.-S. Shao and M. Zaro, The complete NLO corrections to dijet hadroproduction, JHEP 04 (2017) 076, [1612. 06548 ]. 